\title{
Evidence of $d$-phenylglycine as delivering tool for improving l-dopa absorption
}

\author{
Chun-Li Wang ${ }^{1}$, Yang-Bin Fan' ${ }^{1}$, Hsiao-Hwa Lư ${ }^{2}$ Tung-Hu Tsai ${ }^{3}$, Ming-Cheng Tsai ${ }^{4}$, Hui-Po Wang ${ }^{1 *}$
}

\begin{abstract}
Background: I-Dopa has been used for Parkinson's disease management for a long time. However, its wide variety in the rate and the extent of absorption remained challenge in designing suitable therapeutic regime. We report here a design of using $d$-phenylglycine to guard $l$-dopa for better absorption in the intestine via intestinal peptide transporter I (PepT1).

Methods: $d$-Phenylglycine was chemically attached on 1 -dopa to form $d$-phenylglycine-I-dopa as a dipeptide prodrug of I-dopa. The cross-membrane transport of this dipeptide and I-dopa via PepT1 was compared in brushboarder membrane vesicle (BBMV) prepared from rat intestine. The intestinal absorption was compared by in situ jejunal perfusion in rats. The pharmacokinetics after i.v. and p.o. administration of both compounds were also compared in Wistar rats. The striatal dopamine released after i.v. administration of $d$-phenylglycine---dopa was collected by brain microdialysis and monitored by HPLC. Anti-Parkinsonism effect was determined by counting the rotation of 6-OHDA-treated unilateral striatal lesioned rats elicited rotation with (+)-methamphetamine (MA).

Results: The BBMV uptake of $d$-phenylglycine---dopa was inhibited by Gly-Pro, Gly-Phe and cephradine, the typical PepT1 substrates, but not by amino acids Phe or l-dopa. The cross-membrane permeability $\left(\mathrm{Pm}^{*}\right)$ determined in rat jejunal perfusion of $d$-phenylglycine-I-dopa was higher than that of $I$-dopa ( $2.58 \pm 0.14$ vs. $0.94 \pm 0.10)$. The oral bioavailability of $d$-phenylglycine---dopa was 31.7 times higher than that of 1 -dopa in rats. A sustained releasing profile of striatal dopamine was demonstrated after i. v. injection of $d$-phenylglycine-/-dopa $(50 \mathrm{mg} / \mathrm{kg})$, indicated that $d$-phenylglycine-l-dopa might be a prodrug of dopamine. $d$-Phenylglycine---dopa was more efficient than I-dopa in lowering the rotation of unilateral striatal lesioned rats (19.1 $\pm 1.7 \%$ vs. $9.9 \pm 1.4 \%)$.

Conclusion: The BBMV uptake studies indicated that $d$-phenylglycine facilitated the transport of I-dopa through the intestinal PepT1 transporter. The higher jejunal permeability and the improved systemic bioavailability of $d$-phenylglycine-l-dopa in comparison to that of $I$-dopa suggested that $d$-phenylglycine is an effective delivery tool for improving the oral absorption of drugs like I-dopa with unsatisfactory pharmacokinetics. The gradual release of dopamine in brain striatum rendered this dipeptide as a potential dopamine sustained-releasing prodrug.
\end{abstract}

\section{Background}

l-Dopa (Figure 1), a dopamingenic precursor, has long been used for the treatment of Parkinson's disease [1-4]. Clinically use of this drug was reported to have wide range of inter- and intra-patient variations in the rate and the extent of absorption [5,6]. The inconsistent pharmacokinetics remained as the major issue in designing optimal regime in the disease management $[7,8]$. The variation in oral bioavailability due to the

\footnotetext{
* Correspondence: hpw@tmu.edu.tw

'Taipei Medical University College of Pharmacy, 250 Wu-Hsing St., Taipei,

110-31, Taiwan

Full list of author information is available at the end of the article
}

interaction of $l$-dopa with diet protein is, in part, attributed to its complicated absorption through the amino acid transport systems [9-11]. Although many dopamine agonists emerged, $l$-dopa in combination with metabolic enzyme inhibitors is still the first choice for the treatment of Parkinson's disease [2,3].

Recent reports indicated that intestinal PepT1, a member of proton-coupled oligopeptide transporter system, is responsible for the absorption of a variety of diand tripeptide mimetic drugs such as amino- $\beta$-lactams [12-14] and ACE inhibitors [15]. The structure feature of PepT1 substrates was established [16-18] and the

\section{(Ciomed Central}


<smiles>N[C@@H](C(=O)N[C@@H](Cc1ccc(O)c(O)c1)C(=O)O)c1ccccc1</smiles><smiles>N[C@@H](Cc1ccc(O)c(O)c1)C(=O)O</smiles>

Figure 1 The structures of $d$-phenylglycine-I-dopa and I-dopa

transport system has been used in the design of novel oralabsorbable drugs $[19,20]$.

Based on the thought that $d$-phenylglycine is the common moiety in the molecules of PepT1-mediated orally absorbable amino- $\beta$-lactams [21], we thought that this moiety might be useful as a seeing-eye dog for guiding $l$-dopa to transport through the intestine via PepT1. We therefore synthesized a series of $d$-phenylglycinecontaining di- and tripeptide derivatives as dopamine prodrug [22]. Rationale behind the design of these compounds was that the oral bioavailability of $l$-dopa might be improved due to the affinity of $d$-phenylglycine to PepT1. Besides, the fast decarboxylation of $l$-dopa in peripheral circulation might be prevented or prolonged as the free amino group of $l$-dopa is blocked by $d$-phenylglycine.

This report describes the transport of $d$-phenylglycine$l$-dopa via PepT1 by measuring the uptake in brush-border membrane vesicles (BBMV) prepared from rat intestine. The intestinal absorption of this compound and $l$-dopa was compared by measuring the steady-state plasma concentration after in situ jejunal perfusion and by determining the pharmacokinetics after oral administration in rats. Anti-Parkinsonism effects after oral administration of $d$-phenylglycine- $l$-dopa and $l$-dopa were also compared by measuring the change of the $(+)$-methamphetamine induced rotation of dopamine-depleted unilateral striatallesioned rats. Correlation between pharmacological activity and the pharmacokinetic profile was analyzed.

\section{Methods \\ Materials}

Chemicals, reagent grade for synthesis and analytical grade for biological studies, were from Sigma-Aldrich (St. Louis, MO, U.S.A), E. Merck KG (Darmstadt, Germany), Fluka Chemika (Buchs, Switzerland), Acros (NJ, U.S.A) and Wako (Richmond, VA, U.S.A) companies. Acidwashed alumina was purchased from RiedaL-de Haen Company (Spring Valley, CA, U.S.A.). Melting points were determined in Buchi (Flawil, Switzerland) 510 capillary melting point apparatus and were uncorrected. IR spectra were carried out on a Perkin-Elmer (Shelton, CT, U.S.A) $1760 \mathrm{FT}-\mathrm{IR}$ instrument. ${ }^{1} \mathrm{H}$ NMR spectra were determined on a Bruker (Wissem-bourg, France) 80 $\mathrm{MHz}$ or Bruker $400 \mathrm{MHz}$ spectrometer with chemical shifts recorded in parts per million relative to tetramethylsilane. Mass and high-resolution mass (HRMS) were measured on Finnigan (San Jose, CA. U.S.A.) MAT 4510 and JEOL (Boston, MA, U.S.A.) JNS-D300 spectrometer respectively. Branson (Danbury, CT. U.S.A.) Sonifier 450 sonicator, Kubota (Tokyo, Japan) 2010 or Eppendorf AG (Hamberg, Germany) 5415C centrifuge Model 905 incubator (Cherng Huei Instrument Co., Tainan, Taiwan) and Ystral (Ballrechten-Dottingen, Germany) Laboratory series $\times 10 / 20$ homogenizer were used in the preparation of intestinal mucosal suspension. Osmolarity of test solutions was determined with Wescor 5500 vapor pressure osmometer (Wescor Company, Logan, UT, U.S.A.). Male Wistar rats (300 - 350 g) from the Animal center of National Taiwan University were used in preparing intestinal mucosal suspension, BBMV and in perfusion studies. The same species of rats weighing 180 - $200 \mathrm{~g}$ were used in rotational behaviour studies. Male Sprague-Dawley rats (280 - 320 g) were used for determining brain dopamine. Animal studies were in accordance with the National Institute of Health Guide for the Care and Use of Laboratory Animals.

\section{Brush-Boarder Membrane Vesicle (BBMV) Uptake}

The intestinal cross-membrane transport of $d$ phenylglycine- $l$-dopa and $l$-dopa was investigated using simulated intestinal brush-boarder membrane vesicle $[23,24]$. BBMV was prepared using magnesium precipitation method [25]. Protein content was determined. The purity of BBMV was determined by measuring the activity of the marker enzymes, alkaline phosphatase and aminopeptidase. Generally, these two enzymes were enriched 8 21 folds in the preparation. The activity of $\mathrm{Na}^{+}, \mathrm{K}^{+}$ATPase, the marker enzyme of basolateral membrane, was very small. Normal function of BBMV was confirmed by measuring the uptake of $d$-glucose. In the presence of $\mathrm{Na}^{+}$ gradient $\left(\left[\mathrm{Na}^{+}\right]_{\text {in }}<\left[\mathrm{Na}^{+}\right]_{\text {out }}\right)$, an overshoot phenomenon of glucose uptake with peak values of 9-11 times the equilibrium was routinely observed. The membrane vesicles were preloaded in the buffer solution containing $300 \mathrm{mM}$ mannitol and $16 \mathrm{~m} M$ HEPES/Tris (pH 7.4) before the experiment. The uptake of test compounds in BBMV was measured by rapid filtration.

\section{Degradation of Compounds in Intestinal Mucosa Suspension}

Mucosa suspension was prepared from the intestine of male Wistar rats according to established method [26] and was stored in an ice bath before use.

\section{In Situ Rat Perfusion}

Literature procedure was followed for the preparation of perfusion solutions and the jejunal segments [27]. To maximize the absorption and to prevent the test 
compounds from being oxidized during perfusion, the experiments were performed at $\mathrm{pH} 6.0$ with $0.02 \%(\mathrm{w} / \mathrm{v})$ ascorbic acid added as antioxidant and nitrogen gas was bubbled through for $10 \mathrm{~min}$ before each experiment. Perfusion solution was pumped through the jejunal segment at a flow rate of $0.2 \mathrm{ml} / \mathrm{min}$ by a syringe pump (Stoelting, KD Scientific, U.S.A.). The jejunal segment was pre-washed with drug-free buffer for $10 \mathrm{~min}$ before the drug solution was pumped in. Outlet tubing samples were collected every 10 min for 6 collection periods after water and solute transport reached steady-state. The dimensionless membrane permeability $\mathrm{Pm}^{*}$ [28] was measured as indications for the disappearance rate of test compound from the intestine. Plasma samples were withdrawn from carotid artery.

\section{Intravenous and Oral Absorption Experiments}

Rats were fasted for at least $18 \mathrm{~h}$ prior to drug administration. Anaesthesia was induced by i.p. injection of urethane $(0.15 \mathrm{~g} / 100 \mathrm{~g}$ body weight). The rats were put under a heating lamp to maintain body temperature.

\section{Chromatography and Validation of Assay Methods}

The HPLC system used in the assay of biological samples consisted of an autosampler (AS950, Jasco, Tokyo, Japan), a Waters Model 600E solvent delivery pump (Millipore, Milford, MA, USA), a Model LC-4C electrochemical detector with a glassy-carbon electrode (Bioanalytical Systems, Inc., West Lafayette, IN, USA), and an integrator (Macintosh LC II with Macintegrator I). A Nucleosil $^{\circ} 10 \mathrm{SA}$ cationic ion-exchange column $(10 \mu \mathrm{m}$, $300 \times 4.0 \mathrm{~mm}$, Macherey-Nagel, Düren, Germany) with a mobile phase comprising $\mathrm{NaCl}(50 \mathrm{mM})$ and $\mathrm{Na}_{2}-$ EDTA $(1.0 \mathrm{mM})$ in $0.1 \mathrm{M}$ ammonium phosphate buffer $(\mathrm{pH} 2.0)$ at a flow rate of $2.0 \mathrm{ml} / \mathrm{min}$ was used for the elution of the samples. The detection limits of $d$-phenylglycine- $l$-dopa and $l$-dopa were $50 \mathrm{ng} / \mathrm{ml}$ and 25 $\mathrm{ng} / \mathrm{ml}$, respectively. HPLC assay methods were validated by determining the precision and accuracy of intra-day and inter-day analysis of serum standards over a period of 6 days. The coefficients of variation for inter- and intraday assays were less than $15 \%$ for both compounds $(n=6)$.

\section{Pharmacokinetic Analysis}

According to the literature $[29,30]$, the area under the plasma concentration-time profile (AUC) was calculated by log-linear trapezoidal rule. Plasma concentration after i.v. administration of drugs were also fitted to a non-compartment model using PCNONLIN and Akeike's Information Criteria, sum of squared residuals, residual plot and correlation coefficient were use for determination of the compartment model. The residual area after the last observed data point was calculated as $\mathrm{C}_{\text {last }} / \mathrm{k}$, where $\mathrm{C}_{\text {last }}$ is the last observed concentration, and $\mathrm{k}$ is the corresponding terminal rate constant. Terminal half-life $\left(t_{1 / 2}\right)$ was estimated compartment model-independently. The fraction of absorption was calculated according to Equation 1.

$$
\mathrm{BA}=\frac{\frac{\mathrm{AUC}_{\text {oral }} \cdot \mathrm{k}_{\text {oral }}}{\text { dose }_{\text {oral }}}}{\frac{\mathrm{AUC}_{\mathrm{iv}} \cdot \mathrm{k}_{\mathrm{iv}}}{\text { dose }_{\text {iv }}}} \times 100 \%
$$

\section{Brain Microdialysis}

Single dose $d$-phenylglycine- $l$-dopa $(50 \mathrm{mg} / \mathrm{kg}$ in $2.5 \mathrm{~mL}$ of normal saline) was administered i. v. via femoral vein to anesthetized male Sprague-Dawley rats $(280-320 \mathrm{~g})$. The body temperature of the rats was maintained at $37^{\circ} \mathrm{C}$ with a heating pad throughout the experiment. The rat was immobilized in a stereotaxic frame (David Kopf Instruments, Tujunga, CA, USA), the skull was surgically exposed, and a hole was drilled with a trephine into the skull based on stereotaxic coordinates. The brain microdialysis system consisted of a CMA/100 microinjection pump (CMA, Stockholm, Sweden) and a microdialysis probe. The dialysis probes $(3 \mathrm{~mm}$ in length) were made of silica capillary in a concentric design with their tips covered by dialysis membrane (Spectrum, $150 \mu \mathrm{m}$ outer diameter with a cut-off at nominal molecular mass of 13000, Laguna Hills, CA, USA). The probe was placed into right striatum $(0.2 \mathrm{~mm}$ anterior to bregma and $3.2 \mathrm{~mm}$ lateral to midline) and perfused with Ringer's solution (147 mM Na+; $2.2 \mathrm{mM} \mathrm{Ca++;} 4 \mathrm{mM} \mathrm{K+;} \mathrm{pH} \mathrm{7.0)}$ at a flow-rate of $1 \mu \mathrm{l}$ $\min ^{-1}$. The position of each probe was verified at the end of experiments. The dialysates was collected at $10 \mathrm{~min}$ intervals and aliquots of $10 \mu \mathrm{l}$ was assayed by microbore HPLC.

The HPLC system consisted of a pump (BAS PM-80, West Lafayette, IN, USA) and an on-line injector (CMA 160 , Stockholm, Sweden) equipped with a $10 \mu \mathrm{l}$ sample loop, a reversed phase C18 microbore column (particle size $5 \mu \mathrm{m}, 150 \times 1 \mathrm{~mm}$ I.D.; Bioanalytical Systems, West Lafayette, IN, USA) and an EC detector (BAS-4C amperometric) coupled to a glassy carbon working electrode and referenced to a $\mathrm{Ag} / \mathrm{AgCl}$ electrode at $750 \mathrm{mV}$ with a range set at 50 nanoamper. Output data from the detector were integrated via an EZChrom chromatographic data system (Scientific Software, San Ramon, CA, USA). The mobile phase for analyzing striatal dopamine, eluted at a flow rate of $0.05 \mathrm{ml} / \mathrm{min}$, comprised $80 \mathrm{ml}$ acetonitrile, $2.2 \mathrm{~m} M$ sodium 1-octanesulfonate, $14.7 \mathrm{~m} M$ monosodium dihydrogen orthophosphate, $30 \mathrm{mM}$ sodium citrate, $0.027 \mathrm{mM}$ EDTA, and $1 \mathrm{ml}$ diethylamine in one liter double distilled water, adjusted 
to $\mathrm{pH} 3.5$ by orthosphoric acid (85\%). The elute was filtered through a Millipore $0.22 \mu \mathrm{m}$ filter and degassed prior to use.

\section{Rotational Behavior of Rats [31-34]}

Male Wistar rats $(180$ - $200 \mathrm{~g})$ were anesthetized with pentobarbital sodium ( $30 \mathrm{mg} / \mathrm{kg}$ body weight, i.p.) and the heads were fixed in a DaviD-Kopf steric taxic frame. A solution of 6-hydroxydopamine (6-OHDA, $2.00 \mathrm{mg} / \mathrm{ml} \times$ $8 \mathrm{ml}$ ) in saline was infused using Paxinos and Watson coordinates (AP 5.3, L 2.0, H $7.8 \mathrm{~mm}$, [34]) into the unilateral substantia nigra compacta $(\mathrm{SNc})$ of brain with a syringe pump through a 30 gauge stainless steel needle at a flow rate of $2 \mu \mathrm{l} / \mathrm{min}$. After two weeks of recovery period, the 6-OHDA treated rats were placed in a spherical bowl (radius $20 \mathrm{~cm}$ ) and secured by a thoracic harness which was connected to a $486 \mathrm{PC}$ computer for automatic recording of rotation induced by $(+)$-methamphetamine (MA). The rotational behavior of rats was recorded $10 \mathrm{~min}$ after MA treatment (MA in saline, 4.00 body weight $\mathrm{mg} / \mathrm{kg}$ of rat, s.c.). The numbers of turns recorded were defined as the control value $\left(\mathrm{T}_{0}\right)$ for each individual animal. Only animals showing a $T_{0}$ greater than 400 were chosen for further experiments. After two weeks of a wash-out period the animals were subjected to drug treatment. Single dose $(0.051 \mathrm{mmol})$ of each test compound was administered orally to rats 5 min prior to MA treatment $(4.00 \mathrm{mg} / \mathrm{kg}$ body weight, s.c.). The rotation counted for a period of $110 \mathrm{~min}$ starting $10 \mathrm{~min}$ after MA treatment was recorded as $T_{d}$ for each tested rat. The percentage of reduction in rotation for each animal was calculated and presented as $\left(\mathrm{T}_{\mathrm{d}}-\mathrm{T}_{0}\right) / \mathrm{T}_{0} \times 100 \%$.

\section{Data Analysis}

Data analysis were performed on Visual dBase and SPSS/PC+ and were represented as mean \pm SE for $n$ experiments. Treatment differences were evaluated by paired-t test.

\section{Results}

\section{d-Phenylglycine-I-dopa Uptake in BBMV}

The uptake of $d$-phenylglycine-l-dopa in BBMV was measured. Amino acid $l$-Phe or $l$-dopa, dipeptide $l$-Gly- $l$-Pro or $l$-Gly-l-Phe, or cephradine was added for investigating the competition with $d$-phenylglycine- $l$-dopa in BBMV uptake (Figure 2).

\section{Stability of $d$-Phenylglycine-I-dopa in Intestinal Mucosal Suspension}

The stability of $d$-phenylglycine- $l$-dopa in the intestine was determined prior to the intestinal absorption studies. In order to simulate intestinal microclimate $\mathrm{pH}$, the compound was incubated with the intestinal mucosal suspension in a pH 6.5 isotonic buffer solution.

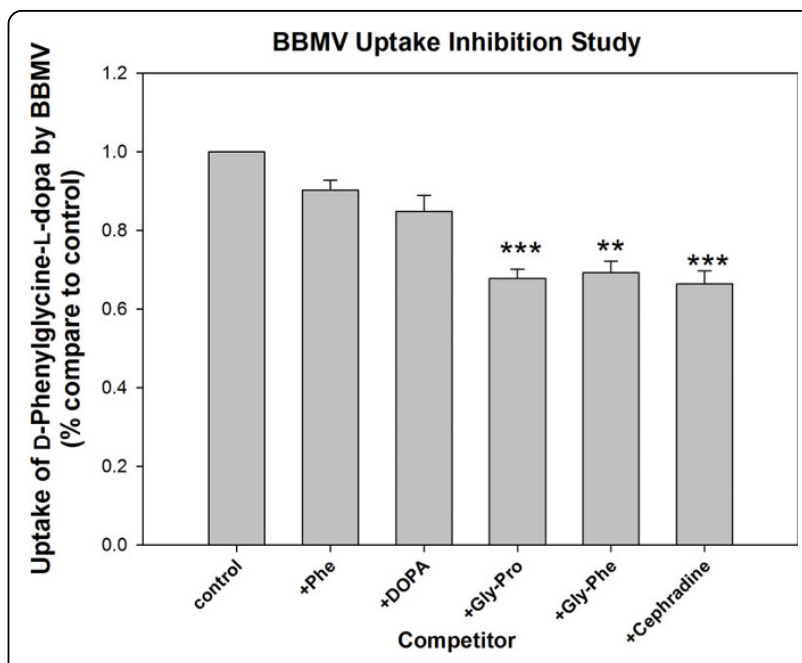

Figure 2 The uptake of $d$-phenylglycine-l-dopa in BBMV with or without the presence of I-Phe, I-dopa, I-Gly-I-Pro, I-Gly-I-Phe and cephradine $\left(* *: p<0.01 ;{ }^{* * *}: p<0.001\right.$.). The BBMV was prepared according to material and methods. The BBMV preparation (20 ml containing approximately $20 \mathrm{mg}$ protein $/ \mathrm{ml}$ ) was added into $200 \mathrm{ml}$ of a reaction buffer (composed of $300 \mathrm{mM}$ mannitol, $25 \mathrm{mM}$ HEPES/Tris buffer $\mathrm{pH} 7.4$, ( $\mathrm{pH}$ was adjusted by adding MES) and the test solution (to $1-2 \mathrm{mM}$ of final conc.) was added. After incubation at room temperature for acquired time, an ice-cold stop solution $(1.5 \mathrm{ml})$ containing $\mathrm{NaCl}(150 \mathrm{mM})$ and HEPES/Tris (16 mM, $\mathrm{pH}$ 7.4) was added and the solution was filtered through a filter paper (Whatman WCN, $0.45 \mu \mathrm{m}$ pore size, $2.5 \mathrm{~cm}$ diameter) under a vacuum. The filter paper was washed twice with $3 \mathrm{ml}$ of the same stop solution. The test compound remained on the filter paper were extracted with $0.5 \mathrm{ml}$ of $0.01 \mathrm{M}$ aqueous $\mathrm{HCl}$ solution by virtue of a vortex motion. The solution $(100 \mu l)$ was injected onto the HPLC column. Test compound bound on the filter paper was determined for correction in different runs using preparations without BBMV added.

$l$-Gly- $l$-Phe comprising essential amino acids degraded rapidly with only $50 \%$ of recovery after $2 \mathrm{~min}$ of incubation. $d$-Phenylglycine- $l$-dopa, on the other hand, was very stable with almost $100 \%$ of recovery after $90 \mathrm{~min}$ of incubation (Figure 3).

\section{Permeability of $\boldsymbol{d}$-Phenylglycine-I-dopa in Rat Intestine}

The absorption of $d$-phenylglycine- $l$-dopa and $l$-dopa was compared in rats by in situ single-pass jejunal perfusion experiments. Amidon's dimensionless crossmembrane permeability $\left(\mathrm{P}_{\mathrm{m}}{ }^{*}\right)$ was determined as a parameter of intestinal absorption [28]. The steady-state plasma concentration was also determined (Table 1).

\section{Pharmacokinetic Profile in Rats}

The mean plasma concentration-time profiles after single dose oral and i.v. administration of $d$-phenylglycine- $l$-dopa and $l$-dopa are depicted in Figure 4. The pharmacokinetic parameters calculated with the data of plasma concentration-time curves based on the non-compartmental model 


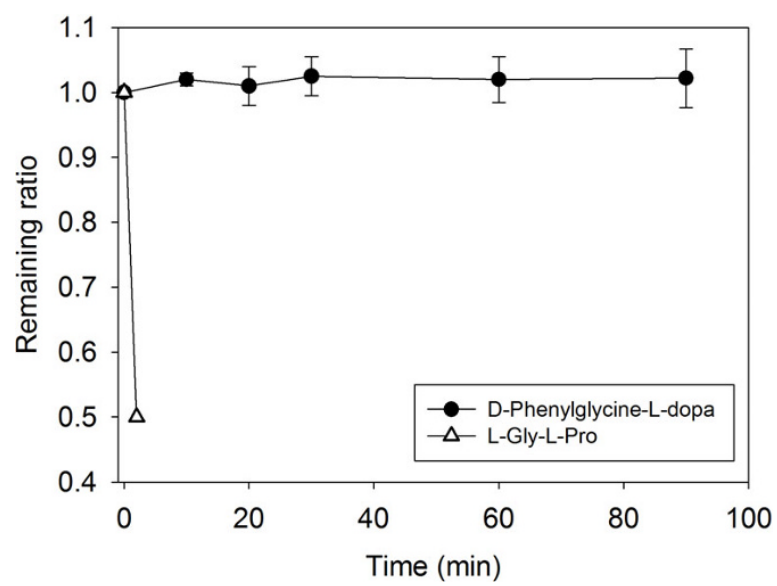

Figure 3 Comparison of the stability of $d$-phenylglycine-I-dopa and I-Gly-I-Phe in rat intestinal mucosa suspension. Each point represents mean $\pm \mathrm{SE}$. of 3 experiments. A methanolic solution (100 $\mu \mathrm{l})$ of the test compound $(1 \mathrm{mg} / \mathrm{ml})$ was diluted with an isotonic mannitol buffer solution ( $\mathrm{pH} 6.5,2.4 \mathrm{ml}$ ) as the stock solution. This stock solution $(1 \mathrm{ml})$ was mixed with the freshly prepared mucosal suspension $(1 \mathrm{ml})$. The mixture was incubated in a water bath at $37^{\circ}$ $\mathrm{C}$ and subjected to sampling at intervals between zero to $90 \mathrm{~min}$ of incubation. Each sampled solution $(200 \mu \mathrm{l})$ was denatured with 0.8 $\mathrm{ml}$ of $\mathrm{MeOH}$ and centrifuged at $6,600 \mathrm{~g}$ for $5 \mathrm{~min}$. Each of the supernatant $(20-100 \mu l)$ was subjected to HPLC assay.

analysis were summarized in Table 2. The fraction of oral absorption (BA) was calculated according to Equation 1. The Striatal dopamine level after i.v. injection of $d$-phenylglycine- $l$-dopa $(50 \mathrm{mg} / \mathrm{kg})$ is depicted in Figure 5.

\section{Anti-Parkinsonism Activity}

The in vivo anti-Parkinsonism effect was determined with conventional rotation model measured in 6-OHDA-treated unilateral striatal-lesioned rats elicited rotation with (+)-methamphetamine (MA) [32,35]. As shown in Table 3, $d$-phenylglycine- $l$-dopa as well as $l$-dopa demonstrated inhibition of MA-induced rotation of rats. With equal molar of test compound administered, the activity of $d$-phenylglycine- $l$-dopa in reducing the rotation of rats was significantly higher than that of $l$-dopa.

\section{Discussion}

The BBMV uptake of $d$-phenylglycine- $l$-dopa was significantly inhibited by dipeptides $l$-Gly-l-Pro $\left.{ }^{* * * * *} p<0.001\right)$,
$l$-Gly-l-Phe $(* * p<0.01)$ and cephradine, a typical PepT1 substrate $(* * *<<0.001)$, while was less inhibited by $l$-Phe and $l$-dopa, suggesting that PepT1 might be involved in the uptake of this dipeptide. We previously reported a kinetic study on the BBMV uptake of $d$-phenylglycine- $\alpha$ methyldopa. The uptake of this dipeptide was also significantly inhibited by typical PepT1 substrate [22]. The high value of Michaelis-Menten kinetic parameter $(\mathrm{Vmax} / \mathrm{Km})$ in comparison to that of passive diffusion $(\mathrm{Kd})$ at low concentrations suggested that PepT1 dominates the transport of the $d$-phenylglycine-containing dipeptide through the intestine. Both results indicated that $d$ phenylglycine increased the intestinal transport of amino acid $\alpha$-methyldopa and $l$-dopa via PepT1.

The absorption of oral drugs in human can be evaluated as dimensionless permeability $\mathrm{P}_{\mathrm{m}}{ }^{*}$ in in situ single-pass perfusion in rats despite the complicated process of absorption in the gastrointestinal tract $[28,36,37]$. The high $\mathrm{P}_{\mathrm{m}}{ }^{*}$ demonstrated by $d$-phenylglycine- $l$-dopa $(2.58 \pm 0.14)$ in comparison to that of $l$-dopa $(0.94 \pm 0.10)$ indicated the high absorption of this dipeptide in the intestine. The steady-state plasma concentration of $d$-phenylglycine$l$-dopa after the perfusion was 31.1 fold, in terms of molar ratio, higher than that of $l$-dopa, indicated that this dipeptide was better absorbed than $l$-dopa.

The pharmacokinetic profiles upon i.v. and oral administration of $d$-phenylglycine- $l$-dopa and $l$-dopa were compared. Although the volume of distribution after i.v. injection of $d$-phenylglycine- $l$-dopa was higher than that of $l$-dopa, this dipeptide was cleared much faster than $l$-dopa from the plasma. This made the systemic bioavailability (AUC) of $d$-phenylglycine- $l$-dopa 7 times lower than that of $l$-dopa $(62.53 \pm 19.68 \mathrm{vs.} 459.81 \pm 195.14 \mathrm{mg} \cdot \mathrm{min} / \mathrm{ml})$. On the contrary, the AUC of $d$-phenylglycine- $l$-dopa was comparable to that of $l$-dopa upon oral administration $(28.85 \pm 8.52$ vs. $27.37 \pm 4.60 \mathrm{mg} \cdot \mathrm{min} / \mathrm{ml})$. As a result, the fraction of oral absorption of $d$-phenylglycine- $l$-dopa was 31 fold higher than that of $l$-dopa $(27.58 \pm 4.56 \%$ vs. $0.87 \pm$ $0.24 \%)$.

The striatal dopamine level increased gradually after i.v. injection of $d$-phenylglycine- $l$-dopa and had not reached plateau 3.5 hours when the anaesthetized mice woke up. The gradual release of dopamine in brain striatum rendered this dipeptide as a dopamine sustained-releasing prodrug.

Table 1 Plasma concentrations of $d$-phenylglycine-I-dopa and I-dopa measured in in situ single-pass jejunal perfusion experiments

\begin{tabular}{ccccc}
\hline Compound & No. of Experiments & $\mathbf{P m}^{*}$ & Blood concentration $(\boldsymbol{\mu g} / \mathbf{m l})$ & $\begin{array}{c}\text { Molar ratio of blood concentration } \\
(\boldsymbol{\mu g} / \mathbf{m l})\end{array}$ \\
\hline $\begin{array}{c}d \text {-phenylglycine-l-dopa } \\
\text { l-dopa }\end{array}$ & 4 & $2.58 \pm 0.14$ & $64.6 \pm 5.40$ & 31.10 \\
\hline
\end{tabular}

${ }^{a}$-dopa was detected only in the plasma sample from one of the three rats tested. It was below detection limit in plasma samples of the other two rats. Data presented are mean \pm SD of $n$ experiments. 


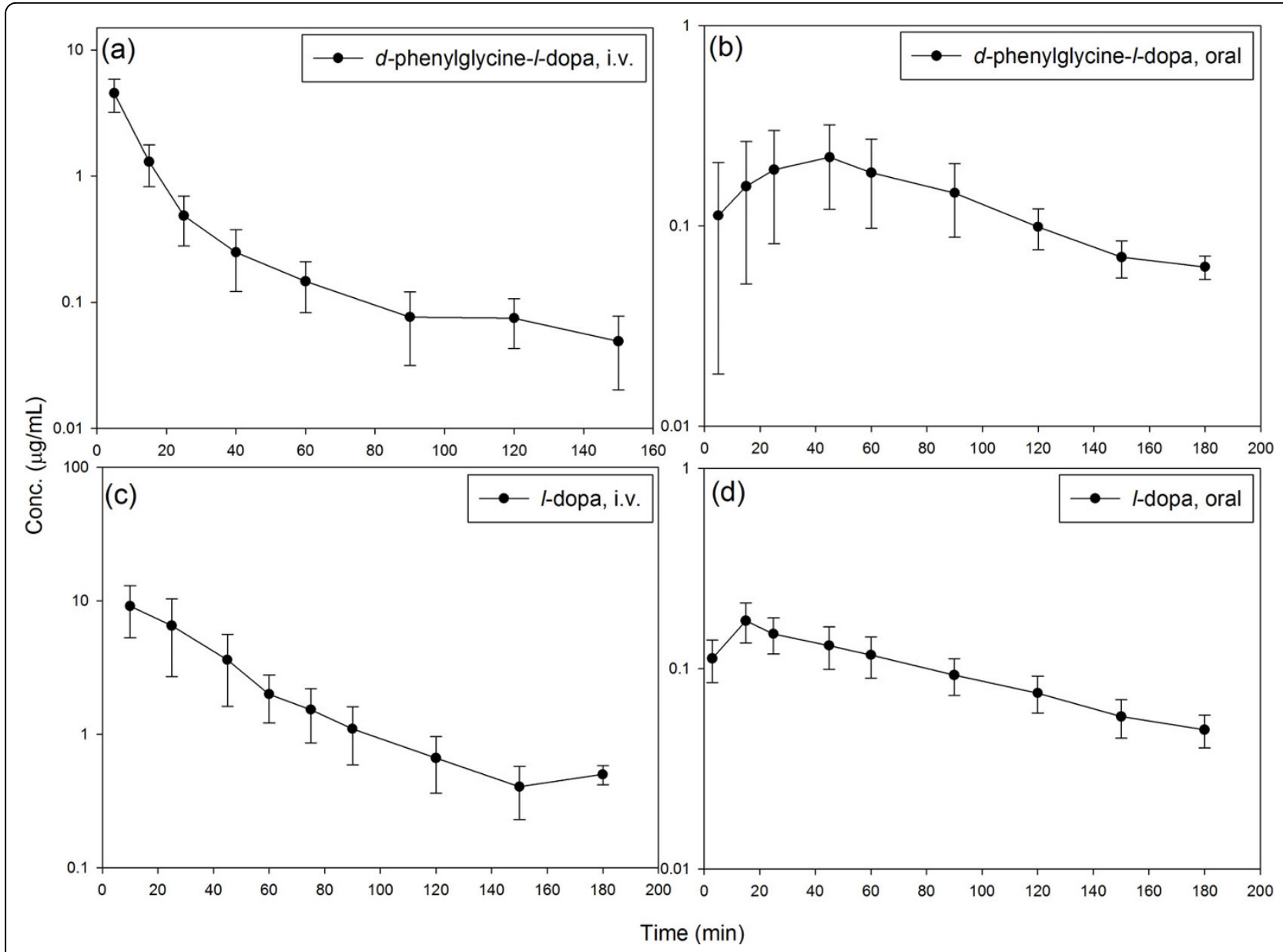

Figure 4 Plasma concentration-time profile of $d$-phenyglycine-I-dopa (a), (b) and I-dopa (c), (d) after i.v. (a), (c) and oral (b), (d) administration in Wistar rats $(\mathbf{n}=\mathbf{6})$. The aqueous solution of test compound with dose equivalent to $5.97 \mathrm{mg} / \mathrm{kg}$ body weight of 1 -dopa was administered either intravenously from the tail vein or orally by a feeding tube. Blood samples were collected from the carotid artery at time intervals of from 1 to $180 \mathrm{~min}$. Heparin sodium (25 I.U./ml in $0.3 \mathrm{ml}$ of saline) was added to blood samples, and were then centrifuged at 6,600 $\mathrm{g}$ for $5 \mathrm{~min}$. Plasma was stored at $-78^{\circ} \mathrm{C}$ until being analyzed. A $200 \mu \mathrm{l}$ of the plasma sample in a $10 \mathrm{ml}$ test tube was mixed with $500 \mu \mathrm{l}$ of 1.0 $M$ Tris buffer ( $\mathrm{pH}$ 8.6, adjusted by EDTA-2 $\mathrm{Na}^{+}$) and $10 \mu \mathrm{l}$ of 3,4-dihydroxybenzylamine (DHBA, $2 \mu \mathrm{g} / \mathrm{ml}$ ) was added as internal standard. Alumina $100 \mathrm{mg}$ was then added and then shake for $15 \mathrm{sec}$ and the supernatant was decanted. The alumina was washed four times with $5 \mathrm{ml}$ of water, and the adsorbed compounds on the alumina was eluted with $200 \mu \mathrm{l}$ of an acidic solution $(0.9 \mathrm{ml}$ of glacial acetic acid in $4.0 \mathrm{ml}$ of $1.0 \mathrm{M}$ phosphate buffer). A $30 \mu \mathrm{l}$ of the eluent was then analyzed by HPLC.

Table 2 Pharmacokinetic parameters derived from non-compartmental analysis after i.v. and oral administration of $d$ phenylglycine-l-dopa and $I$-dopa in rats (mean $\pm S D, \mathbf{n}=6$ )

\begin{tabular}{ccccc} 
& \multicolumn{2}{c}{$\boldsymbol{d}$-phenylglycine-I-dopa } & \multicolumn{2}{c}{ I-dopa } \\
& i.v. & Oral & i.v. & Oral \\
\hline$A \cup C(\mathrm{mg} \cdot \mathrm{min} / \mathrm{ml})$ & $62.53 \pm 19.68$ & $28.85 \pm 8.52$ & $459.81 \pm 195.14$ & $27.37 \pm 4.60$ \\
$\mathrm{t}_{1 / 2}(\mathrm{~min})$ & $254.10 \pm 73.05$ & $142.50 \pm 23.71$ & $101.52 \pm 27.74$ & $184.80 \pm 46.20$ \\
$\mathrm{Cl}_{p}(\mathrm{l} / \mathrm{kg} / \mathrm{min})$ & $0.18 \pm 0.06$ & $0.29 \pm 0.10$ & $0.02 \pm 0.02$ & $0.01 \pm 0.00$ \\
$\mathrm{Vd}_{\mathrm{ss}}(\mathrm{l} / \mathrm{kg})$ & $11.01 \pm 5.08$ & $35.7 \pm 17.1$ & $1.22 \pm 0.89$ & $1.22 \pm 0.36$ \\
$t_{\max }(\mathrm{min})$ & - & $38.30 \pm 17.72$ & - & $25.02 \pm 16.10$ \\
Fraction of absorption $(\%)$ & - & $27.58 \pm 4.56$ & - & $0.87 \pm 0.24$ \\
\hline
\end{tabular}




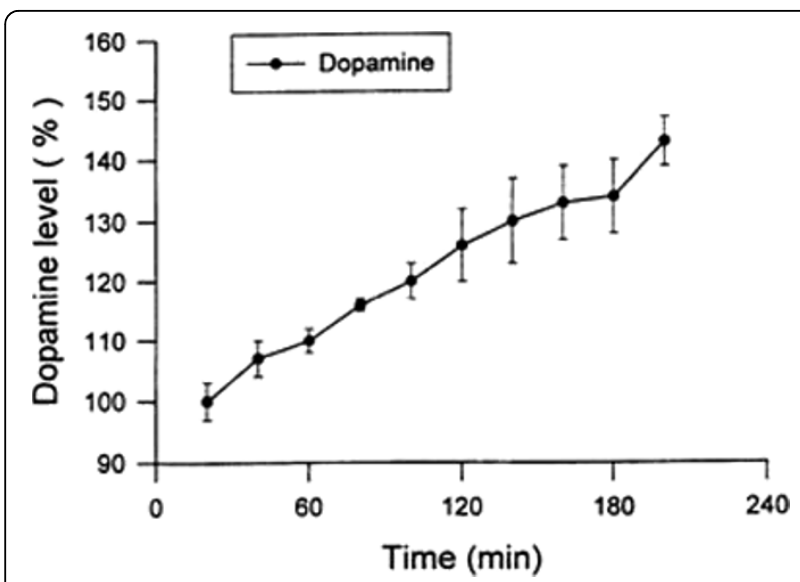

Figure 5 Striatal dopamine level after i. v. injection of $d$ phenylglycine-l-dopa. Values represent the group mean \pm s.e.m. (n $=4$ ). Single dose $d$-phenylglycine-l-dopa (50 mg/kg in $2.5 \mathrm{~mL}$ of normal saline) was administered i.v. via femoral vein to anesthetized male Sprague-Dawley rats $(280$ - $320 \mathrm{~g})$. The dialysates collected from the brain microdialysis probe was subjected to HPLC to measure the striatal dopamine concentration at $10 \mathrm{~min}$ interval.

$d$-Phenylglycine- $l$-dopa after oral administration demonstrated higher activity than $l$-dopa in reducing the MA-induced rotation in rats with statistical significance $(19.1 \pm 1.7 \%$ vs. $9.9 \pm 1.4 \%, * * * 0<0.001)$, suggesting its anti-Parkinsonism activity. Whether the activity came from the dipeptide per se or from the released dopamine needs further investigation. Correlation between the pharmacological activity and the pharmacokinetic parameters indicated that the high activity demonstrated by $d$-phenylglycine- $l$-dopa might partially come from its better oral absorption.

\section{Conclusion}

$d$-Phenylglycine-l-dopa was proved to be better absorbed from the intestine than $l$-dopa. The BBMV uptake suggested that $d$-phenylglycine might act as a seeing-eye dog for guiding $l$-dopa to transport through the intestine via intestinal PepT1 oligopeptide transporter. The higher anti-Parkinsonism activity of this dipeptide in comparison to that of $l$-dopa might come from the improved oral bioavailability. The pharmacokinetic

Table 3 Percent reduction of (+)-methamphetamineinduced rotation in unilateral nigrostriatal-lesioned rats after oral administration of $d$-phenylglycine-l-dopa and I-dopa

\begin{tabular}{cccc}
\hline Compound & $\begin{array}{c}\text { dose } \\
(\mathbf{m g} / \mathbf{k g})\end{array}$ & $\begin{array}{c}\text { no. of } \\
\text { experiment }\end{array}$ & $\begin{array}{c}\text { Reduction in rotation } \\
\text { (mean } \pm \text { SE \%) }\end{array}$ \\
\hline $\begin{array}{c}\text { d-phenylglycine- } \\
\text { l-dopa }\end{array}$ & 16.7 & 6 & $19.1 \pm 1.7^{*}$ \\
l-dopa & 10.0 & 6 & $9.9 \pm 1.4$ \\
\hline
\end{tabular}

profile of striatial dopamine indicated that $d$-phenylglycine $l$-dopa might be useful as a slow dopamine-releasing prodrug for therapeutic use. The improved intestinal permeability with improved oral bioavailability as a consequence, suggested the potential use of $d$-phenylglycine as an effective delivery tool for drugs with unsatisfied oral absorption.

\section{Abbreviations}

PepT1: Intestinal peptide transporter T1; BBMV: Brush-boarder membrane vesicle; MA: Methamphetamine

\section{Acknowledgements}

This study was supported by grant NCS95-2320-B-039-049-MY3 of National Science Council (2008) and DOH99-TD-C-111-008 of the Department of Health, the Republic of China.

\section{Author details}

${ }^{1}$ Taipei Medical University College of Pharmacy, 250 Wu-Hsing St., Taipei, 110-31, Taiwan. ${ }^{2}$ Roche Products Ltd., Taipei, Taiwan. ${ }^{3}$ Institute of Traditional Medicine, School of Medicine, National Yang-Ming University, 155 Li-Nong Street, Section 2, Taipei, Taiwan. ${ }^{4}$ Department of Pharmacology, College of Medicine, National Taiwan University, No. 1, Section 1, Jen-Ai Rd., Taipei, Taiwan.

\section{Authors' contributions}

CLW and YBF carried out PK and rat rotational studies and drafted the manuscript. HHL carried out permeability studies. MCT designed rat rotational behavior studies. THT carried out the brain microdialysis studies. HPW conceived and is responsible for the study. All authors read and approved the final manuscript.

\section{Competing interests}

The authors declare that they have no competing interests.

Received: 13 July 2010 Accepted: 6 September 2010

Published: 6 September 2010

\section{References}

1. Birkmayer $W$, Hornykiewicz $O$ : The effect of $/$-3,4-dihydroxyphenylalanine (= DOPA) on akinesia in Parkinsonism. Parkinsonism Relat Disord 1998, 4:59-60.

2. Hauser R: Levodopa: Past, present and future. Eur Neurol 2009, 62:1-8.

3. LeWitt PA: Levodopa for the treatment of Parkinson's disease. N Eng J Med 2008, 359:2468-2476.

4. Olanow CW, Stern MB, Sethi K: The scientific and clinical basis for the treatment of Parkinson's disease. Neurology 2009, 72(Suppl 4):S1-S136.

5. Juncos JL: Levodopa: pharmacology, pharmacokinetics, and pharmacodynamics. Neurol Clin 1992, 10:487-509.

6. Nomoto M, Nishikawa N, Nagai M, Yabe H, Nakatsuka A, Moritoyo H, Moritoyo T, Kubo M: Inter- and intra-individual variation in I-dopa pharmacokinetics in the treatment of Parkinson's disease. Parkinsonism Relat Disord 2009, 15(Suppl 1):S21-S24.

7. Jenner P: Molecular mechanisms of $I$-dopa -induced dyskinesia. Nat Rev Neurosci 2008, 9:665-677.

8. Abbruzzese G: Optimising levodopa therapy. Neurol Sci 2008, 29 S377-S379.

9. Lennernas H, Nilsson D, Aquilonius SM, Ahrenstedt O, Knutson L, Paalzow LK: The effect of I-leucine on the absorption of levodopa, studied by regional jejunal perfusion in man. Br J Clin Pharmacol 1993, 35:243-250.

10. Kempster PA, Wahlqvist ML: Dietary factors in the management of Parkinson's Disease. Nutr Rev 1994, 52:51-58.

11. Crevoisier C, Zerr P, Calvi-Gries F, Nilsen T: Effects of food on the pharmacokinetics of levodopa in a dual-release formulation. Eur J Pharm Biopharm 2003, 55:71-76. 
12. Groneberg DA, Döring F, Eynott PR, Fischer A, Daniel H: Intestinal peptide transport: ex vivo uptake studies and localization of peptide carrier PEPT1. Am J Physiol Gastrointest Liver Physiol 2001, 281:G697-G704.

13. Brodin B, Nielsen CU, Steffansen B, Frokjaer S: Transport of peptidomimetic drugs by the intestinal di/tri-peptide transporter, PepT1. Pharmacol Toxicol 2002, 90:285-296.

14. Hironaka T, Itokawa S, Ogawara K, Higaki K, Kimura T: Quantitative evaluation of PEPT1 contribution to oral absorption of cephalexin in rats. Pharm Res 2009, 26:40-50.

15. Knütter I, Wollesky C, Kottra G, Hahn MG, Fischer W, Zebisch K, Neubert RH, Daniel H, Brandsch M: Transport of angiotensin-converting enzyme inhibitors by $\mathrm{H}+$ /peptide transporters revisited. J Pharmacol Exp Ther 2008, 327:432-41.

16. Brandsch $M$, Knutter L, Leibach FH: The intestinal $\mathrm{H}+$ /peptide symporter PEPT1: structure-affinity relationships. Eur J Pharm Sci 2004, 21:53-60.

17. Vig BS, Stouch TR, Timoszyk JK, Quan Y, Wall DA, Smith RL, Faria TN: Human PEPT1 pharmacophore distinguishes between dipeptide transport and binding. J Med Chem 2006, 49:3636-3644.

18. Larsen SB, Jørgensen FS, Olsen L: QSAR models for human $\mathrm{H}^{+} /$peptide symporter, hPEPT1: Affinity prediction using alignment-independent descriptors. J Chem Inf Model 2008, 48:233-241.

19. Brandsh M: Transport of drugs by proton-coupled peptide transporters: pearls and pitfalls. Expert Opin Drug Metab Toxicol 2009, 5:887-905.

20. Majumdar S, Duvvuri S, Mitra AK: Membrane transporter/receptor-targeted prodrug design: Strategies for human and veterinary drug development. Adv Drug Deliv Rev 2004, 56:1437-1452.

21. Wang HP, Lu HH, Lee JS, Cheng CY, Mah JR, Hsu WL, Yen CF, Lin CJ, Kuo HS: Intestinal absorption studies on peptide mimetic alphamethyldopa prodrugs. J Pharm Pharmacol 1996, 48:270-276.

22. Wang HP, Wang CL: Biological Transporters as Target for New Drug Design. J Exp Clin Med 2010, 1:31-38.

23. Sheikh MI, Muller JV: Preparation and use of renal and intestinal plasma membrane vesicles for toxicological studies. In Biochemical Toxicology: A Practical Approach. Edited by: Snell K, Mullock B. IRL press, Oxford; , 1 1987:153-182.

24. Anand BS, Patel J, Mitra AK: Interactions of the dipeptide ester prodrugs of acyclovir with the intestinal oligopeptide transporter: competitive inhibition of glycylsarcosine transport in human intestinal cell line-Caco2. J Pharmacol Exp Ther 2003, 304:781-791

25. Okano $\mathrm{T}$, Inui $\mathrm{K}$, Takano M, Hori R: $\mathrm{H}^{+}$gradient-dependent transport of aminocephalosporins in rat intestinal brush-border membrane vesicles. Biochem Pharmacol 1986, 35:1781-1786.

26. Lu HH, Thomas J, Fleisher D: Influence of $d$-glucose-induced water absorption on rat jejunal uptake of two passively absorbed drugs. $J$ Pharm Sci 1992, 81:21-25.

27. Hu M, Subramanian P, Mosberg HI, Amidon GL: Use of the peptide carrier system to improve the intestinal absorption of $l$-alpha-methyldopa: Carrier kinetics, intestinal permeabilities and in vitro hydrolysis of dipeptidyl derivatives of $I$-alpha-methyldopa. Pharm Res 1989, 6:66-70.

28. Amidon GL, Sinko PJ, Fleisher D: Estimating human oral fraction dose absorbed: A correlation using rat intestinal membrane permeability for passive and carrier-mediated compounds. Pharm Res 1988, 5:651-654.

29. Reigelman S, Collier P: The application of statistical moment theory to the evaluation of in vivo dissolution time and absorption time. $J$ Pharmacokinet Biopharm 1980, 8:509-534.

30. Kaplan SA, Jack ML, Cotler S, Alexander K: Utilization of area under the curve to elucidate the disposition of an extensively biotransformed drug. J Pharmacokinet Pharmacodyn 1973, 1:201-216.

31. Paxinos G, Waston C: The Rat Brain in Sterotaxic Coordinates New York: Academic Press, 21986.

32. Hudson JL, van Horne CG, Stromberg I, Brock S, Clayton J, Masserano J, Hoffer BJ, Gerhard GA: Correlation of apomorphine and amphetamine induced turning with nigrostriatal dopamine content in unilateral 6hydroxydopamine lesioned rats. Brain Res 1993, 626:167-174.

33. lancu R, Mohapel P. Brundin P. Paul G: Behavioral characterization of a unilateral 6-OHDA-lesion model of Parkinson's disease in mice. Behav Brain Res 2005, 162:1-10.

34. Beal MF: Experimental models of Parkinson's disease. Nat Rev Neurosci 2001, 2:325-332.
35. Metz GA, Tse A, Ballermann M, Smith LK, Fouad K: The unilateral 6-OHDA rat model of Parkinson's disease revisited: an electromyographic and behavioural analysis. Eur I Neurosci 2005, 22:735-744.

36. Lennernäs $\mathrm{H}$ : Animal data: The contribution of the Ussing chamber and perfusion system to predicting human oral drug delivery in vivo. Adv Drug Deliv Rev 2007, 59:1103-1120.

37. Johnson DA, Amidon GL: Determination of intrinsic membrane transport parameters from perfused intestine experiments: a boundary layer approach to estimating the aqueous and unbiased membrane permeabilities. J Theor Biol 1988, 131:93-106.

doi:10.1186/1423-0127-17-71

Cite this article as: Wang et al:: Evidence of $d$-phenylglycine as delivering tool for improving l-dopa absorption. Journal of Biomedical Science 2010 17:71.

\section{Submit your next manuscript to BioMed Central and take full advantage of:}

- Convenient online submission

- Thorough peer review

- No space constraints or color figure charges

- Immediate publication on acceptance

- Inclusion in PubMed, CAS, Scopus and Google Scholar

- Research which is freely available for redistribution

Submit your manuscript at www.biomedcentral.com/submit
Ciomed Central 